\title{
Das SIWF ist gut positioniert
}

\author{
Geschäftssitzung, Informationsplattform, Austausch- und Diskussionsforum, Impuls- \\ geberin: Die Plenarversammlung des Schweizerischen Instituts für ärztliche Weiter- \\ und Fortbildung SIWF erwies sich auch 2014 als Veranstaltung mit vielen Facetten. \\ Den Delegierten und Gästen, den Teilnehmenden wurde ein umfassender Einblick \\ in die Aktivitäten und Tätigkeitsfelder des SIWF geboten.
}

Bruno Kesseli

«In science the credit goes to the man who convinces the world, not to the man to whom the

\begin{abstract}
SIWF-Präsident Werner Bauer konnte zu Beginn der Versammlung auf ein kleines und zwei grössere Jubiläen hinweisen. Das kleine betraf das SIWF selbst, das in diesem Jahr bereits sein fünfjähriges Bestehen feiert - mit Werner Bauer als Präsidenten. Dieser beliess es dabei, das Jubiläum den Anwesenden zur Kenntnis zu bringen, kündigte aber immerhin für das «Zehnjährige» ein Feuerwerk an Festlichkeiten an - diesem Ereignis darf man also mit einiger Spannung entgegenblicken. Jubiläen grösseren $\mathrm{Zu}$ schnitts konnten dagegen zwei «Urgesteine» feiern, die seit «Ewigkeiten» mit grossem Engagement in den Diensten des SIWF beziehungsweise dessen Vorgängerin $\mathrm{KWFB}^{*}$ stehen: Abteilungsleiterin Petra Bucher ist seit 20, Geschäftsführer Christoph Hänggeli gar seit 25 Jahren dabei (siehe Kasten). Das Plenum würdigte diese Treue mit einem Applaus.
\end{abstract}

\section{idea first occurs.» (Osler I)}

* Kommission für Weiter- und Fortbildung der FMH

** Sir William Osler (1847-1919), kanadischer Mediziner und Humanist mit grossem Einfluss auf die Entwicklung der medizinischen Weiterbildung.

bkesseli[at]emh.ch

\section{Mitteilungen des Präsidenten}

Verschiedene Aktivitäten, Veranstaltungen und Projekte des SIWF in diesem Jahr wurden vom Präsidenten im Sinne eines kleinen Tour d'horizon präsentiert - und wie üblich kam auch Sir William Osler** ausgiebig zum Zug (siehe eingestreute Zitate).

\section{Projektförderung}

Die SIWF-Projektförderung ist ein neues Instrument zur Verbesserung der ärztlichen Weiterbildung [1]. Durch die finanzielle Unterstützung von ausgewählten Weiterbildungsprojekten will das SIWF zur Verbesserung der Weiterbildung und zur Entlastung der Weiterbildungsverantwortlichen beitragen. In diesem Jahr wurden aus 62 Einreichungen vier Projekte ausgewählt und mit insgesamt 125000 Franken unterstützt [2]. «Es war eine schwierige Auswahl, aber wir haben unser Geld sicher gut investiert», kommentierte Werner Bauer die ersten Erfahrungen mit der Projektförderung.

\section{SIWF-Award}

Mit dem SIWF-Award werden Ärztinnen und Ärzte für besonderes Engagement in der Weiterbildung ausgezeichnet. Die Nomination erfolgt durch ehemalige Assistenzärztinnen und -ärzte. Wie Werner Bauer betonte, geht es dabei nicht um eine Rangierung, sondern um eine Möglichkeit für Assistentinnen und Assistenten, ehemaligen Weiterbildnern ihre Anerkennung auszudrücken. 2014 wurden 19 Weiterbildende ausgezeichnet [3].

\section{MedEd Symposium}

Ebenfalls eine Premiere war das MedEd Symposium des SIWF vom 11. September 2014 im Kultur Casino Bern. «MedEd» steht für «Medical Education», und die gut besuchte Veranstaltung brachte denn auch eine interessante und anregende Mischung von Beiträgen zu den «Perspektiven der ärztlichen Bildung». Die Liste der Referenten und Podiumsgäste umfasste

\section{Plenarversammlung des SIWF}

Das Schweizerische Institut für ärztliche Weiter- und Fortbildung SIWF stellt in über 120 Fachgebieten eine qualitativ hochstehende Weiter- und Fortbildung der Ärztinnen und Ärzte sicher. Es vereinigt als autonomes Organ der FMH alle wesentlichen Akteure und Organisationen im Bereich der Weiterund Fortbildung.

Die jährliche Plenarversammlung des SIWF erfüllt verschiedene Funktionen. Zum einen bilden die Plenumsmitglieder ein wahl- und beschlussfähiges Gremium, das in Analogie zur Ärztekammer Geschäfte aus seinem Zuständigkeitsbereich behandelt. Andererseits ist die Versammlung, zu der auch Gäste verschiedener Provenienz eingeladen werden, eine Art Forum, das themenspezifische Inputs vermittelt, dem Erfahrungsaustausch unter den Teilnehmenden dient und Diskussionen aller Art ermöglicht. 


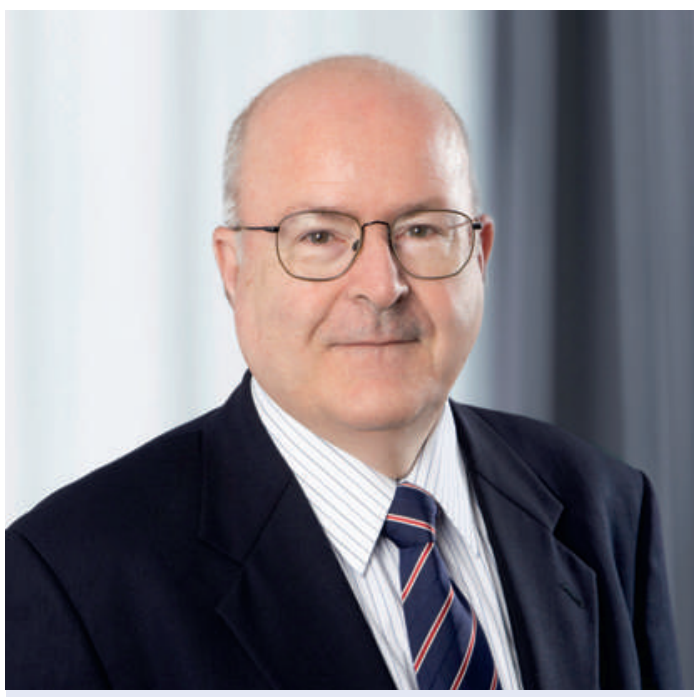

Seit 5 Jahren am Steuer des SIWF: Werner Bauer.

neben Pascal Strupler, dem Direktor des Bundesamts für Gesundheit, eine Reihe namhafter Experten aus Medizin, Spitalverwaltung, Gesundheitsökonomie, Politik und Behörden, die das Thema aus unterschiedlicher Perspektive beleuchteten und für angeregte Diskussionen sorgten.

\section{Allgemeine Lernziele}

Mit allgemeinen Lernzielen sind unspezifische Kompetenzen bzw. Fähigkeiten zum selbstorganisierten Handeln gemeint, die es Ärztinnen und Ärzten ermöglichen, ihre Patienten optimal zu betreuen. Wie Werner Bauer erläuterte, sollen die «Allgemeinen Lernziele» vom Sorgenkind zum Projekt werden. Unterstützt wird dieser Prozess von Dr. Jean Biaggi, der auf diesem Gebiet über grosse Erfahrung verfügt. Geplant ist zunächst eine Umfrage, um die Bedürfnisse der verschiedenen Akteure zu evaluieren. In der Folge sollen die Projektziele konkretisiert werden.

\section{Faculty Development Workshops des Royal College of Physicians}

Das Echo auf die 2012 in Luzern erstmals durchgeführten Workshops des Royal College of Physicians waren so positiv, dass sich eine Fortsetzung nachgerade aufdrängte. Mit den Kursen wurde ein Angebot für Ober- und Chefärzte geschaffen, das diese darin unterstützen sollte, ihren Lehr- und Führungsaufgaben besser gerecht zu werden. Die Erfahrungen hätten indes gezeigt, so Werner Bauer, dass gerade auch jüngere Kolleginnen und Kollegen stark von diesen Workshops profitieren könnten.

\section{Arbeitsplatzbasierte Assessments}

Unter dem Begriff «Arbeitsplatzbasierte Assessments» (AbAs) wird die strukturierte Beobachtung der Assistenten mit Feedback durch ihre Weiterbildner verstanden. Sprach Werner Bauer bei den «Allge- meinen Lernzielen» noch von einem «Sorgenkind», griff er bei den AbAs gar zur Metapher des «unerwünschten Kinds». Allerdings betraf diese Umschreibung nur noch die Vergangenheit, denn mittlerweile seien die AbAs an den meisten Weiterbildungsstätten zur Selbstverständlichkeit geworden.

\section{Steuerung der Weiterbildung?}

BAG-Direktor Pascal Strupler hatte am 1. MedEdSymposium unter anderem das Thema der Steuerung der Weiterbildung aufgegriffen. Die Frage, wie viele Ärzte aus welchen Fachgebieten es in den einzelnen Versorgungsregionen brauche, sei hochkomplex, meinte Werner Bauer dazu. Ausserdem gebe es immer eine Latenzzeit, bis sich Massnahmen auswirkten. Die Erfahrungen in Ländern wie Frankreich, die die Weiterbildung sehr dirigistisch steuer-

«The greater the ignorance, the greater the dogmatism.» (Osler II)

ten, seinen nicht überzeugend. Das Thema werde das SIWF sicher weiter beschäftigen, zumal es in der «Plattform ärztliche Bildung» von BAG und GDK weit oben in der Agenda stehe.

\section{Wahlen Vorstandsmitglieder}

Der Vorstand des SIWF umfasst maximal 19 Mitglieder. 14 von ihnen werden von den medizinischen Fakultäten sowie ärztlichen Berufsverbänden und Fachgesellschaften ex officio in den Vorstand delegiert. Die restlichen fünf Mitglieder sind von der Plenarversammlung aus den eigenen Reihen frei wählbar. Für diese fünf Sitze stellten sich sechs Kandidaten zur Wahl, die allesamt in den ersten beiden Wahlgängen das absolute Mehr der Stimmen erreichten. Vor dem dritten Wahlgang, in dem die Stimmenzahl pro Kandidat ausschlaggebend gewesen wäre, zog Professor Luigi Mariani von der Schweizerischen Gesellschaft für Neurochirurgie seine Kandidatur zurück. Gewählt wurden Tiziano Cassina (bisher), Gieri Cathomas (bisher), Jürg Hafner (bisher), Susanne Stöhr (bisher) und Urs von Wartburg (neu). Der Bestätigungswahl zu stellen hatte sich schliesslich SIWF-Vizepräsident JeanPierre Keller, dem von den Delegierten per Akklamation das Vertrauen für eine weitere Amtszeit ausgesprochen wurde.

\section{Resultate der Facharztbefragung 2013}

Professor Michael Siegrist vom Institute for Environmental Decisions der ETH Zürich stellte in seinem Referat ausgewählte Resultate der Facharztbefragung 2013 vor. Erhoben wurde die Zufriedenheit der Absolventen einer Facharztweiterbildung nach 3 und 
4 Jahren. Die Rücklaufquote der schriftlichen, per Post verschickten Befragung betrug 48,2 Prozent, was sehr beachtlich ist. Zusammenfassend kann festgehalten werden, dass sich die Mehrheit der Befragten durch ihre Weiterbildung gut auf den Alltag als Facharzt vorbereitet fühlt. Der Anteil derjenigen, die sich schlecht vorbereitet fühlen, liegt unter 10 Prozent. Auch die Qualität der Weiterbildung wird mehrheitlich als gut beurteilt. Trotz der grundsätzlichen Zufriedenheit mit der Weiterbildung bestehen deutliche Unterschiede zwischen den einzelnen Facharztrichtungen. Zudem werden auffallend häufig subjektiv erlebte Defizite in den Bereichen Kommunikation, ökonomische Grundlagen, Umgang mit Fehlern und Festlegung der Arbeitsfähigkeit angegeben. Der Referent gab seiner Hoffnung Ausdruck, dass die Resultate der Studie die Diskussion stimulieren, wenn es um die Überarbeitung des Lernzielkatalogs geht. Diese Erwartung ging insofern gleich in Erfüllung, als seine Ausführungen eine Reihe lebhafter und auch kritischer Kommentare aus den Reihen der Delegierten auslösten.

\section{Strategie SIWF}

Die Strategie SIWF wurde im Rahmen der letztjährigen Plenarversammlung verabschiedet [4]. Werner Bauer betonte, dass es sich dabei nicht um eine Hochglanzbroschüre für die Ablage, sondern um einen Leitfaden für zukunftsorientiertes Arbeiten handle, der immer wieder auf seine Aktualität überprüft werde. Zentral sei die Frage, «wie wir unser System weiterentwickeln, weil sich die Welt eben ändert». Ärztliche Organisationen hätten eine Tendenz

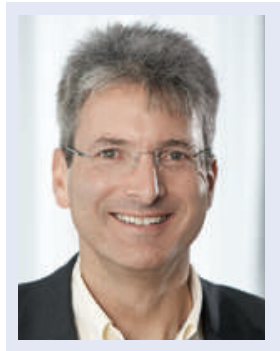

Ein Jubilar ...

Seit 25 Jahren laufen die Fäden im SIWF beziehungsweise vor 2009 im Sekretariat Aus-, Weiter- und Fortbildung AWF bei Christoph Hänggeli zusammen. Der Jurist trat 1989 in den Rechtsdienst der FMH ein und übernahm 1997 die Leitung des Sekretariats AWF, das 2008 unter seiner Mitwirkung in die Geschäftsstelle SIWF überführt wurde. Im Verlauf seiner Tätigkeit für FMH und SIWF hat Christoph Hänggeli unzählige Projekte betreut und erfolgreich umgesetzt. So koordinierte und begleitete er die Arbeiten rund um die Ausarbeitung der ersten FMH-Standesordnung, die mit der Verabschiedung an der Ärztekammer im Dezember 1996 zum Abschluss gebracht wurde. Grossprojekte wie Strukturreform (1989), neue Weiterbildungsordnung (1992) und Fortbildungsordnung (1995) wurden ebenfalls von ihm betreut. Seit 2002 erteilt die KWFB* der FMH respektive das SIWF (ab 2009) eidgenössische Facharzttitel - eine weitere Herausforderung für Christoph Hänggeli und sein Team. Dazu sind die Weiterbildungsprogramme bereits zum zum Bewahren. Dies habe zwar durchaus positive Aspekte, genüge im heutigen gesundheitspolitischen Umfeld aber nicht: «Wir Ärzte müssen proaktiv sein, wir können nicht nur mauern und stoppen.» Als wichtige Subziele nannte er unter anderem das Design einer eigenen Marke, das Abklären der Notwendigkeit und sinnvoller Möglichkeiten einer Steuerung der fachärztlichen Versorgung sowie die Optimierung der Lernzielfestlegung und Evaluation der Weiterbildung.

\section{Revision Weiterbildungsordnung (WBO)}

Die Delegierten hatten im Rahmen des diesjährigen Plenums darüber zu befinden, ob die Erteilung von Fähigkeitsausweisen (FA) und Schwerpunkttiteln (SP) auch in Zukunft an die Mitgliedschaft bei der FMH gekoppelt sein sollte. Die Anhörung der zuständigen FMH-Gremien hatte ergeben, dass auch der Zentralvorstand der FMH die Empfehlung aussprach, die Mitgliedschaftspflicht abzuschaffen. Vor diesem Hintergrund beantragte der Vorstand dem Plenum, die Mitgliedschaftspflicht bei FA und SP aufzuheben und die entsprechenden Bestimmungen in der Weiterbildungsordnung zu revidieren. Dieser Antrag wurde von den Delegierten mit 53 Ja- gegen 8 Nein-Stimmen angenommen.

Weitere Beschlusstraktanden betrafen die Revision des Weiterbildungsbildungsprogamms Allgemeine Innere Medizin, die Revision der Fortbildungsordnung und die Revision des Fähigkeitsprogramms Akupunktur - Traditionelle Chinesische Medizin. Die Anträge des Vorstands wurden in allen drei Fällen klar angenommen. Ein längere Diskussion löste dage-

zweiten Mal vom Eidgenössischen Departement des Innern akkreditiert worden und die nächste Akkreditierung steht für 2018 an. Trotz der permanent hohen Belastung blieb Christoph Hänggeli noch Zeit für die persönliche Weiterbildung: 2005 schlosser das berufsbegleitend absolvierte Nachdiplomstudium «Executive Master of Public administration MPA» der Universität Bern erfolgreich ab.

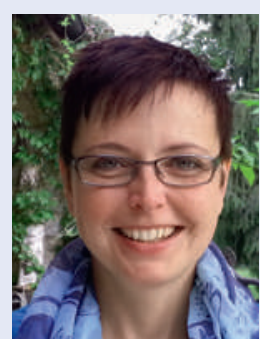

... und eine Jubilarin Petra Bucher ist vielen Ärztinnen und Ärzten als kompetente Ansprechpartnerin seit zwei Jahrzehnten ein Begriff, früher noch unter dem $\mathrm{Na}$ men «Bäriswyl», wie Werner Bauer in seiner Laudatio festhielt. Sie begann ihre Laufbahn vor 20 Jahren im Sekretariat der Abteilung Aus-, Weiter- und Fortbildung, aus dem sie schon bald nicht mehr wegzudenken war. Mittlerweile ist sie Bereichsleiterin im Sekretariat SIWF, das Ärztinnen und Ärzten, Institutionen und Behörden als Anlaufstelle in allen Belangen der ärztlichen Weiterund Fortbildung dient. 
gen der Antrag zur Schaffung eines neuen Fähigkeitsausweises «Endovenöse thermische Ablation von Stammvenen bei Varikose» aus. Auch dieser Antrag fand aber schliesslich mehrheitlich Zustimmung.

\section{e-Logbuch und Fortbildungsplattform}

Dass das e-Logbuch, in dem Assistenzärztinnen und -ärzte ihre Weiterbildung online dokumentieren können, grundsätzlich ein nützliches Hilfsmittel ist, wird mittlerweile in Ärztekreisen kaum mehr bestritten. Die Komplexität der Weiterbildungsprogramme, die fehlende Standardisierung bei den Lernzielen sowie grosse Anstrengungen zur Verbesserung der Benutzer- wurde dem Publikum von Professor Pascal O. Berberat geboten, der in der Leitung des Medizindidaktischen Zentrums für Ausbildungsforschung und Lehre der Technischen Universität München tätig ist. Wissen, so einer seiner zentralen Punkte, könne heute weitgehend selbständig erworben werden. Wissen reiche aber (auch) für Ärztinnen und Ärzte nicht aus, es brauche Fähigkeiten. Bei der Anwendung des Wissens sei das «Teaching» nach wie vor essentiell. Berberat plädierte für eine humanistisch geprägte Berufsauffassung, die allerdings nicht mit einer Vernachlässigung oder gar Negierung der naturwissenschaftliche Basis der Medizin einhergehen dürfe.

\section{«The teacher's life should have three periods, study until twenty-five, investigation until forty, profession until sixty.» (Osler III)}

freundlichkeit mit entsprechendem Supportaufwand haben erhebliche Mehrkosten gegenüber den budgetierten Beträgen generiert. Die involvierten Mitarbeiterinnen, deren Aufwand enorm ist, sind auf die Unterstützung der Fachdelegierten angewiesen. In der Diskussion zeigte sich, dass seitens der Fachgesellschaften und Weiterbildner noch viele Fragen offen sind. Das Bemühen, diese in konstruktiver Weise zu lösen, war jedoch von beiden Seiten spürbar.

Bei der Fortbildungsplattform funktioniert die erste Ausbaustufe mittlerweile stabil. Es ist problemlos möglich, ein Fortbildungsprotokoll zu führen und bei erfüllten Bedingungen das Fortbildungsdiplom auszudrucken. Nach Genehmigung durch die Fachgesell-
Zum Schluss gab Werner Bauer nochmals explizit den Fachgesellschaften das Wort. Jean-Michel Gaspoz, Präsident der SGIM, Pierre Valon, Präsident der SGPP, Hansjakob Furrer, Chefarzt Infektiologie a.i. am Inselspital Bern, PD Dr. med. Stefan Duewell, Institutsdirektor Radiologie, Spital Thurgau AG und Jürg Hafner, Leitender Arzt Dermatologie am Universitätsspital Zürich brachten in pointierter Form Gedanken zum Stand und den Perspektiven der Weiterbildung in ihrem Umfeld zum Ausdruck. Die angeregte Diskussion zeigte, dass die Absicht des Präsidenten, die Plenarversammlung (auch) zu einem Austauschforum mit den Fachgesellschaften zu machen, weitgehend realisiert werden konnte.

\section{«The fact is security can only be achieved through constant change, adapting old ideas that have outlived their usefulness to current facts.»}

(Osler IV)

schaften wird dieses automatisch auf www.doctor fmh.ch publiziert, womit auch die Besitzstandwahrung gewährleistet ist. Die zweite Ausbaustufe ist für 2015 vorgesehen und wird ein Register der Fortbildungsveranstaltungen und eine Vereinfachung der Administration für alle Beteiligten bringen.

Der Vorstand stellte den Antrag, dass bis Ende 2015 sämtliche Fachgesellschaften die Möglichkeit anbieten müssen, das Fortbildungsdiplom in ihrem Fachbereich über die Fortbildungsplattform SIWF zu erwerben. Die Delegierten stimmten diesem Antrag zu.

\section{Gastreferat und Austausch mit den Fachgesellschaften}

Ein engagiertes Referat zu aktuellen Entwicklungen und Herausforderungen in der ärztlichen Bildung

\section{Referenzen}

1 Umfassende Informationen dazu in folgendem Artikel, der im SÄZ-Archiv (www.saez.ch $\rightarrow$ Archiv) auch online zugänglich ist: Bauer W, Hänggeli C. SIWF-Projektförderung: ärztliche Weiterbildung unterstützen. Schweiz Ärztezeitung. 2013;94(45):1701-2.

2 Eine Übersicht über die geförderten Projekte findet sich in folgendem Artikel: Kesseli B, Zwyssig I. Bestehende Probleme analysieren und mögliche Lösungsansätze aufzeigen. Schweiz Ärztezeitung. 2014;95(42):1558-60.

3 Die Zusammenstellung der Weiterbildenden, die 2014 ausgezeichnet wurden, ist in den Artikel [2] integriert.

4 Zur Strategie SIWF siehe: Kesseli B. Das SIWF als Kompetenzzentrum für die ärztliche Bildung in der Schweiz positionieren. Schweiz Ärztezeitung. 2014;95(8):296-9. 